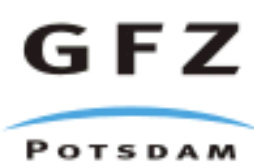

Originally published as:

Wardinski, I., Holme, R., Asari, S., Mandea, M. (2008): The 2003 geomagnetic jerk and its relation to the core surface flows. - Earth and Planetary Science Letters, 267, 3-4, 468-481,

DOI: 10.1016/j.epsl.2007.12.008. 


\title{
The 2003 geomagnetic jerk and its relation to the core surface flows
}

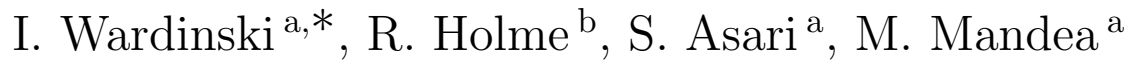 \\ ${ }^{a}$ GeoForschungszentrum Potsdam, Section 2.3: Earth's Magnetic Field, Potsdam, \\ Germany \\ ${ }^{\mathrm{b}}$ University of Liverpool, Department of Earth and Ocean Sciences, Liverpool, UK
}

\begin{abstract}
In this paper we examine the core surface flow obtained by an inversion of a continuous model of the geomagnetic field and its temporal variation using the diffusion-less induction equation. The continuous CHAOS model is derived from satellite data up to spherical harmonic degree 14 and covers the period 1999 to 2006. The CHAOS secular variation, when downward continued to the core surface, shows stripe-like features, which can be attributed to spherical harmonic degree 12 and higher. These contributions are removed by applying a tapering method, and the resulting tapered model is then inverted for the core surface flow. Satellite-based field models have a high spatial resolution; however, their temporal resolution is limited. In order to enhance the temporal resolution of the flow, we additionally constrain the flow to fit the secular variation from ground-based observatory data.
\end{abstract}

A range of solutions, subject to different constraints, are computed, two flow hypotheses being considered: purely toroidal flow and tangentially geostrophic flow. We show that both flow types provide similar results; however, the purely toroidal flow provides a better fit to the secular variation in the equatorial region than 
the tangentially geostrophic flow. We then analyze the residuals between observed secular variation and its predictions from the flow. We note larger residuals for the tangentially geostrophic flow, where strong radial secular variation and a weak radial field are observed. Although diffusive effects can not be ruled out as a potential source of the mismatch, we attribute the larger residuals to be caused by a flawed estimation of the poloidal flow.

We also seek to relate temporal changes in the fluid flow to the geomagnetic jerk which occurred at the beginning of 2003. It is found that this geomagnetic jerk coincides with variations in the zonal flow components of both flow types, suggesting a possible link to torsional oscillations. However, we argue that other flow components are important to obtain a detailed fit of the observed secular variation. Finally, we compare observed changes in the length-of-day and the predictions from the flow solutions.

Key words: Geomagnetism, Core surface flow, Geomagnetic secular variation, Geomagnetic jerk

PACS: 91.25.L-, 91.25.lf

\section{Introduction}

In the last eight years the geomagnetic field generated in the core (main field) and its temporal variation (secular variation) have been probed by three satellite missions: the Ørsted mission, launched in 1999 [32], the CHAMP mission, launched in 2000 [39] and the SAC-C mission launched in 2000. All satellites have been orbiting the Earth in low altitudes $(400-800 \mathrm{~km})$ on nearly

* Corresponding author.

Email address: ingo@gfz-potsdam.de (I. Wardinski). 
polar tracks and have provided globally distributed high-quality vector measurements of the Earth's magnetic field. These data have allowed modelling of the secular variation with high spatial resolution, continuously over the period of the satellite missions. Recently, this led to the satellite-only based CHAOS model [33] which is derived from CHAMP, Ørsted and SAC-C data, covering the time interval from March 1999 to December 2005. This model describes continuously the geomagnetic main field and its secular variation up to spherical harmonic degree 14 between 2000 and 2006.

One intriguing feature of the secular variation, better characterized by recent satellite missions, is the 2003 geomagnetic "jerk", a discontinuity in the second time derivative of the main field. This event was detected in CHAMP satellite data and the CHAOS model [34]. It also appears to be present in geomagnetic observatory data. Fig. 1 shows the X (North), Y (East) and Z (vertical down) components of Niemegk and Kakioka observatory. Unlike other geomagnetic jerks, which are clearly visible in the secular variation of the $\mathrm{Y}$ component of the field, this jerk is more pronounced in the $\mathrm{Z}$ component. [34] analyzed the spatial scale of this event on the basis of secular acceleration maps (cf. their Fig. 9). It was shown that the spatial extent of the jerk is rather limited. The maximum jerk strength is found near $107^{\circ} \mathrm{E}, 17^{\circ} \mathrm{S}$ and minimum at $77^{\circ} \mathrm{E}$ and $34^{\circ} \mathrm{N}$, respectively. These extrema coincide with extrema of the secular acceleration.

Recently, [9] suggested that geomagnetic jerks might be caused by torsional oscillations. These oscillations within the liquid outer core are expected to occur when the geomagnetic dynamo operates in a Taylor state [12; 41], i.e. when the magnetic field is configured so that the axial Lorentz torque vanishes when integrated over cylindrical surfaces aligned with the rotation axis. 

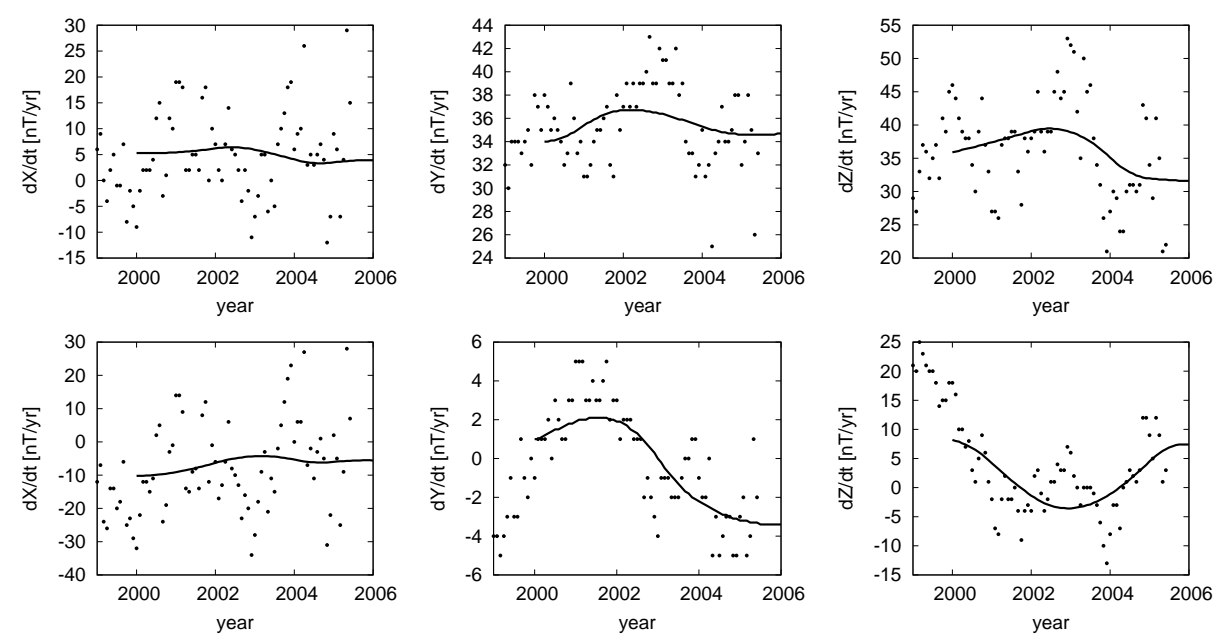

Fig. 1. Comparisons of the observed secular variation (dots) at Niemegk observatory (top) and Kakioka (bottom) and as determined from the CHAOS model (solid line). Deviations from this state cause rigid-body azimuthal accelerations in order to balance the Lorentz torque between adjacent cylindrical annuli, where the typical periods of the oscillation may be some decades and shorter $[12 ; 13]$. Variations of the magnetic field have been used to model the core surface flow $[11 ; 15]$ and equatorially symmetric zonal flow has been interpreted in terms of torsional oscillations $[37 ; 48 ; 49]$. The variations of the core angular momentum derived from the equatorially symmetric zonal flow correlate well with the observed variations in length-of-day (LOD) [28; 29; 37], suggesting that torsional oscillations are important to explain decadal LOD variations. Moreover, [23] provided evidence that geomagnetic jerks coincide with sharp changes in the rate of change of LOD.

A first demonstration that time changes in the flow is needed to explain jerks was given by [17]. Here, we derive the core surface motion by inverting a modified CHAOS model and using the frozen flux assumption. We seek to relate temporal changes in the fluid flow with the geomagnetic jerk occurred in 2003. Two flow types, a purely toroidal and a tangentially geostrophic flow 
are evaluated. However, we limit our analysis to the toroidal flow component, for two reasons. First, both flow assumptions provide estimates of the toroidal flow component, allowing convenient comparison. The second reason relies on considerations how magnetic diffusion works at the CMB. A possible diffusion mechanism is flux expulsion, involving the poloidal advection of toroidal field $[1 ; 7 ; 18]$; if this process acts, then the estimation of the poloidal flow, using the frozen flux assumption, is likely to be flawed. In section 2 we discuss some properties of the CHAOS model and a treatment to reduce noisy contributions from the high-degree main field and secular variation. We also describe additional observatory data sets used to constrain the flow solutions. The third section summarizes the methodology used to model the core surface flow from secular variation. In the next section we discuss the results of our fluid flow inversion and possible sources of errors, particularly, possible errors due to disregarding magnetic diffusion in the inversion. Finally, the temporal variability of the flows is considered and the relative core angular momentum from the flow is derived and compared with the observed LOD.

\section{Secular variation from CHAOS}

Before inverting the CHAOS model for the core surface flow, we examine its properties at the core mantle boundary (CMB). The map of the radial secular variation shows maxima along the equator, which can be attributed to spherical harmonic degree 12 and higher (Fig. 2). This may become more clear from the power spectrum $W(l)$ of the mean-square secular variation which is given by

$$
W(l)=(l+1)\left(\frac{a}{c}\right)^{(2 l+4)} \sum_{m=0}^{l}\left(\left(\dot{g}_{l}^{m}\right)^{2}+\left(\dot{h}_{l}^{m}\right)^{2}\right)
$$




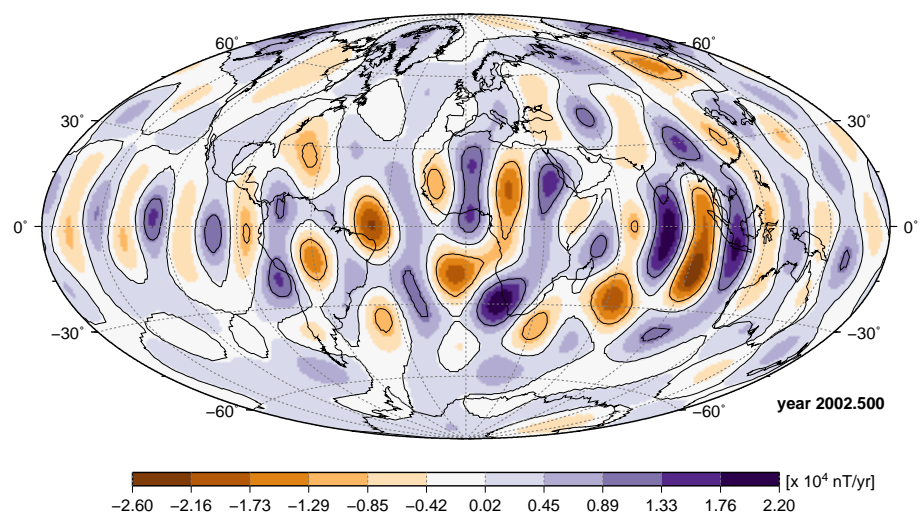

Fig. 2. Map of the radial secular variation at the CMB computed from the CHAOS model.

where $a$ and $c$ are the Earth's and core radii, $l$ and $m$ the spherical harmonic degree and order, respectively. In Fig. 3 the temporally averaged spectrum is plotted against spherical harmonic degree, where spectra for epochs from 1999 to 2006, separated by one year interval are averaged. The vertical bars represent the standard deviation of the power for single degrees and reflect the temporal variability. The spectrum of the secular variation at the CMB is "blue" (power increases with harmonic degree), and [25; 43] discuss the possible reasons for this. In addition, however, the effects of model uncertainty increase strongly with harmonic degree, due to downward continuation of the effects of surface noise. This leads to decreasing reliability of the secular variation estimates with increasing degree. Olsen (pers. comm.) suggests that the detailed values of the secular variation above degree 12 should be treated with caution. Another possible source of error could be the use of scalar satellite data at high latitudes. It is well known that scalar data do not well resolve sectorial terms because of the Backus effect [3; 4], but other sources like smallscale and time variable induced fields may contribute to uncertainties.

In order to reduce the noise signatures a tapering method [44] is applied to 


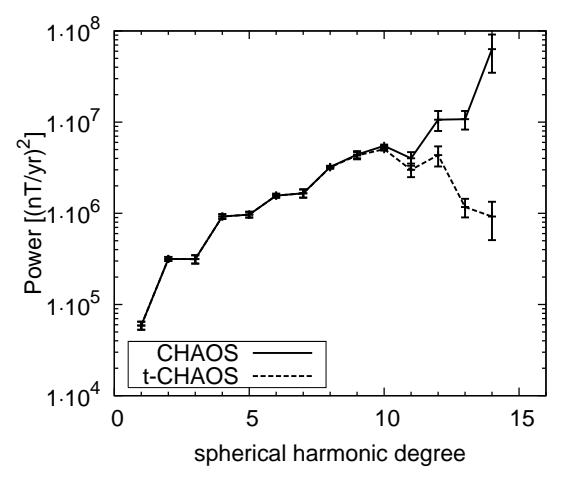

Fig. 3. The averaged spectra of the secular variation at the CMB for the original and tapered CHAOS model over the period 2000 - 2006, respectively. The vertical bars indicate the temporal variance of the spectral power for a given degree.

the secular variation and the field

$$
\dot{g}_{l}^{m}=\frac{\dot{g}_{l 0}^{m}}{1+\lambda\left(\frac{a}{c}\right)^{(2 l+4)}} .
$$

The $\dot{g}_{l}^{m}$ are the tapered coefficients and the $\dot{g}_{l 0}^{m}$ of the original secular variation model. A similar relation applies to the $\dot{h}_{l}^{m}, g_{l}^{m}$ and $h_{l}^{m}$. The high-degree coefficients are controlled by the tapering and the degree of fall-off is determined by the damping parameter $\lambda$.

The appropriate damping parameter $\lambda$ is chosen by visual inspection of maps, when the zebra-like features of radial secular variation have been eliminated; our damping choice significantly reduces these contributions above degree 12 . We denote our tapered modification of the original CHAOS model t-CHAOS. Other damping schemes would be possible; an alternative more severe damping scheme would be to set all spectral contributions above degree 12 to zero (Olsen, pers. comm.). As an alternative, we could assign very high variances to the poorly-constrained high-degree secular variation terms. However, while the flow models produced would be essentially unchanged from our approach, comparisons of secular variation fit (by maps) would be rendered meaningless 


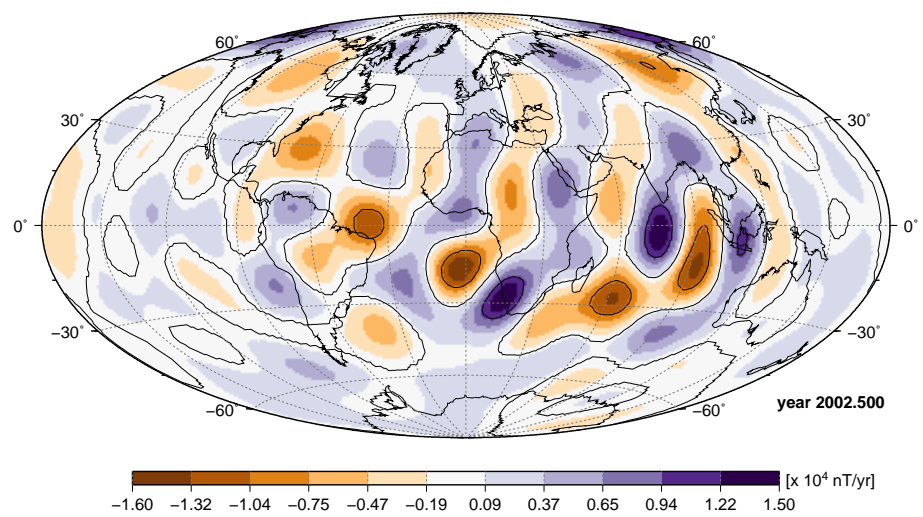

Fig. 4. Map of the radial secular variation at the CMB computed from the tapered CHAOS model.

by the high-degree noise, and hence we prefer the tapering scheme adopted.

Fig. 3 shows that, as required, the spectral power of the tapered model is reduced on shorter wavelengths. A map of the tapered radial secular variation (Fig. 4) demonstrates, however, that some distinct features of the radial secular variation at the $\mathrm{CMB}$ remain after tapering, such as a strong patch under South Africa and a cluster of patches under Indian and the Indonesian regions (see Fig. 4). The radial secular variation in the Pacific region is rather weak, consistent with earlier reports $[26 ; 42]$.

\section{Modelling the core surface flow}

The determination of the core surface flow is briefly described in this section (for more details see $[11 ; 27 ; 28]$ ). We assume that over the period covered by the CHAOS model the observed secular variation is entirely determined by the advection of the magnetic field, and so neglect magnetic diffusion. This is the frozen flux assumption [40]. To estimate the fluid motion at the core 
surface the radial component of the diffusion-less induction equation is used

$$
\dot{\mathrm{B}}_{\mathrm{r}}=-\nabla_{h} \cdot\left(\mathrm{vB}_{\mathrm{r}}\right)
$$

where $B_{r}$ is the radial component of the magnetic field, $\dot{B}_{r}$ the radial secular variation, $\mathbf{v}$ the fluid velocity at the CMB and $\nabla_{h}$ the horizontal divergence. [2] showed that for the frozen flux assumption to hold some conditions must be satisfied and that further assumptions must be applied to reduce the ambiguity of flow determination. In this study we focus on two assumptions. The first assumption applied to reduce the non-uniqueness of flow inversion is the tangentially geostrophic assumption. It consists in the dynamical supposition that Coriolis and pressure gradient forces within the liquid outer core drive the fluid motion $[21 ; 30]$ and it provides the constraint

$$
\nabla_{h} \cdot(\mathbf{v} \cos \theta)=0
$$

where $\theta$ is the colatitude. The second assumption is that the flow is purely toroidal, i.e. that $\nabla_{h} \cdot \mathbf{v}=0$. Then, the flow parallel to $\nabla_{h} \mathrm{~B}_{\mathrm{r}}$ can be estimated by

$$
\mathbf{v}=-\frac{\dot{\mathrm{B}}_{\mathrm{r}}}{\left|\nabla_{h} \mathrm{~B}_{\mathrm{r}}\right|},
$$

except at places where $\nabla_{h} B_{r}=0$ [46]. The purely toroidal assumption implies that motions are confined to a spherical surface, i. e. no up- or downwelling appear in a compositionally or thermally stratified layer close to the core surface. Although there is some observational evidence for a stably stratified layer at the top of the liquid outer core [14], the existence of such a layer is still a matter of debate [19]. The tangentially geostrophic constraint is stronger, because toroidal non-uniqueness applies everywhere, whereas tangential geostrophy only becomes invalid in the equatorial region, where the pressure gradient force can no longer be balanced by the Coriolis force, and also it fails to re- 
solve the flow non-uniqueness along closed iso-lines of $\mathrm{B}_{\mathrm{r}} / \cos \theta[5]$. However, as reported by [25], the purely toroidal flow does not show strong signs of non-uniqueness when compared with the non-uniqueness contours.

The flow is decomposed into toroidal and poloidal components

$$
\mathbf{v}=\mathrm{v}_{\mathrm{tor}}+\mathrm{v}_{\mathrm{pol}}=\nabla_{h} \times T \hat{\mathbf{r}}+\nabla_{h} r S
$$

$S$ and $T$ are scalars which are expanded in Schmidt-normalized real spherical harmonics in space and cubic B-splines in time (similar to [27]).

Flow solutions are sought to represent large scale flows and are truncated at spherical harmonic degree 14, although, small scale flows, up to degree 28, are able to generate secular variation up to degree 14. The flow harmonics are constrained to converge by this limit. This is ensured by minimizing the norm of the second spatial derivatives of the flow components [8]. High degree secular variation is retained in the inversion, requiring a maximum degree of 42 , but these degrees are constrained to be small, otherwise strong small scale secular variation is generated. Solutions have to be smooth in their temporal variation and the knots of the spline interpolation are spaced as in CHAOS. The solution minimizes the objective function $\Phi$,

$$
\Phi(m)=(\mathbf{y}-\mathbf{A m})^{\top} \mathbf{C}_{\mathbf{e}}{ }^{-1}(\mathbf{y}-\mathbf{A m})+\mathrm{m}^{\top} \mathbf{C}_{\mathbf{m}}{ }^{-1} \mathrm{~m},
$$

where $\mathbf{A}$ is an operator which relates the model $\mathbf{m}$ to the data $\mathbf{y} . \mathbf{y}-\mathbf{A m}$ is the misfit between data and model prediction, subject to the regularization. $\mathbf{C}_{\mathbf{e}}$ and $\mathbf{C}_{\mathbf{m}}$ are the error and model covariance matrix, respectively. $\mathbf{C}_{\mathbf{m}}$ is an expression of all quadratic norms (geostrophic, spatial, temporal and observatory misfit) which represent our a-priori information on the model properties. The data are weighted by the error covariance matrix $\mathbf{C}_{\mathbf{e}}$ which accounts for 
the error of the Gauss coefficients. Its diagonal elements are set to

$$
\mathbf{C}_{\mathbf{e}}=\frac{1}{l+1} \mathbf{I}
$$

where $\mathbf{I}$ is the identity matrix and $l$ the spherical harmonic degree. This matrix is defined by the condition of minimizing the mean square secular variation integrated over the Earth's surface [31]:

$$
\iint_{a}\left(\dot{\mathbf{B}}(\mathbf{v})-\dot{\mathbf{B}}_{\mathrm{CHAOS}}\right)^{2} d S d t
$$

where $\dot{\mathbf{B}}(\mathbf{v})$ is the prediction of the secular variation from the flow.

The appropriate spatial and temporal regularization is chosen by considering a trade-off curve between r.m.s. secular variation misfit and the mean flow velocity. The optimal solution is chosen at the "knee" of the curve ([similar to 37]). A similar scheme is applied to find the solution of the purely toroidal flow.

Following [22] flow solutions are sought also to minimize the misfit to secular variation at observatory sites:

$$
\sum_{i}\left(\dot{\mathbf{B}}_{i}(\mathbf{v})-\dot{\mathbf{B}}_{i o b s}\right)^{2} / \sigma_{i}^{2}
$$

where $\dot{\mathbf{B}}_{i}(\mathbf{v})$ is the prediction of secular variation at the observatory sites and $\dot{\mathbf{B}}_{i o b s}$ are annual first differences derived from observatory monthly means of the three field components X, Y and Z [44]. This condition is controlled by a Lagrange multiplier and enters $\mathbf{C}_{\mathbf{m}}$ of (7). The set of observatories used consists of about 103 observatories throughout the period 2000 to 2005. The data are obtained from the World Data Center Edinburgh ${ }^{1} \cdot \sigma_{i}^{2}$ are the errors of data, assumed to be correlated for each single observatory, but to be uncorre-

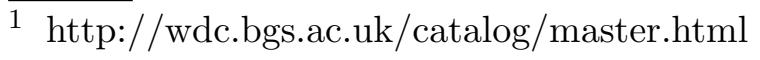


lated between observatories (cf. [44]). This approach provides more confidence in our results than fitting only the model.

\section{Core surface flow from t-CHAOS}

Details of the flow solutions and the damping parameters of the preferred solutions are summarized in Table A.1. TG refers to the tangentially geostrophic solution and PT to the purely toroidal flow.

Before discussing the results in more detail, we mention here a general result. The flow solutions constrained to fit the observatory secular variation differ little from those without this constraint. However, the secular variation predictions differ when computed from each solution. Fig. 5 shows secular variation predictions of the inversion constrained to fit or not observatory secular variation. The predictions of the unconstrained inversion are, apart from an offset identical to the t-CHAOS secular variation, whereas the predictions of the constrained inversion, in most cases, show finer temporal details.

Table A.1 shows that the solutions are largely comprised of toroidal and geostrophic ingredients which mean a general similarity of the flow maps, Fig.6. Both flow solutions are highly symmetric with respect to the equator, between 72 and $77 \%$ of the kinetic flow energy. Asymmetric flows generate the gyres under Labrador, Siberia and Southern Indian Ocean, respectively. Some distinctions exist, for instance the gyre under Labrador, visible in the tangentially geostrophic flow, is not clear in the purely toroidal flow. Further, the southerly motion of the tangentially geostrophic flow under Siberia is displaced to the west in the purely toroidal solution, where this jet is part of 

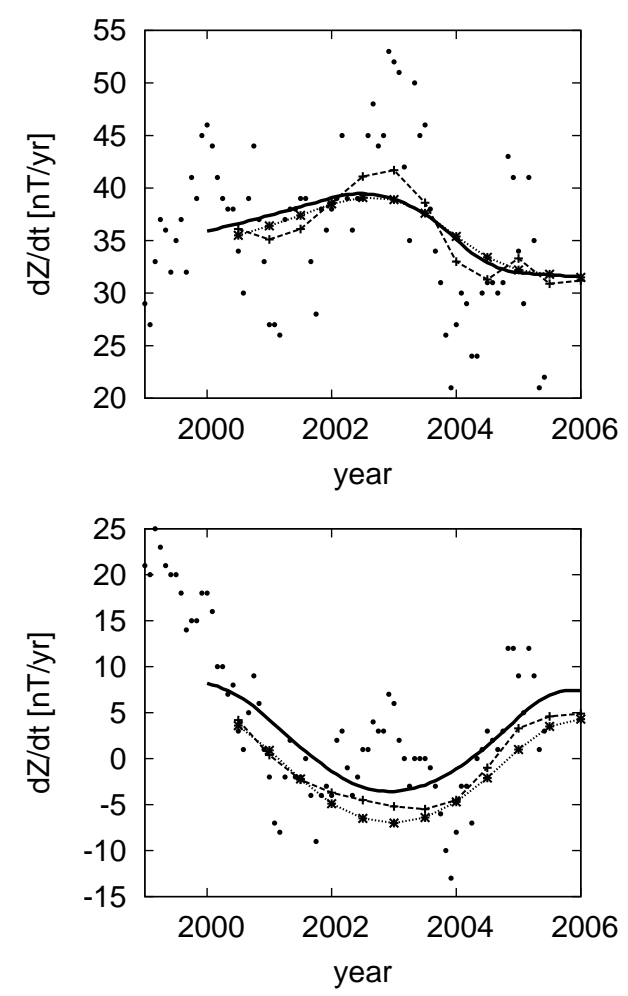

Fig. 5. Comparisons of the observed radial secular variation (dots) at Niemegk observatory (top) and Kakioka (bottom) and as determined from the t-CHAOS model (solid line), the prediction of the inversion constrained to fit observatory data (dashed line), and the prediction of a t-CHAOS-only inversion (doted lines).

a great gyre of the Northern Hemisphere with nearly no fluid motion under Europe. As a consequence of the different constraints, the PT flow has no upand downwelling, whereas no equator-crossing motion exists in the TG flow. Fig. 7 shows the fluid up- and down-welling of the TG flow: blue patches correspond to regions of downwelling motion and red patches indicate upwelling motion. Intense up- and down-welling stands out in the region south of India.

Our TG solution is similar only at large scale to [26], whereas narrow scale features are rather dissimilar. Like the tangentially geostrophic solution of [25], our TG flow has strong motion under Indonesia and narrow scale fea- 

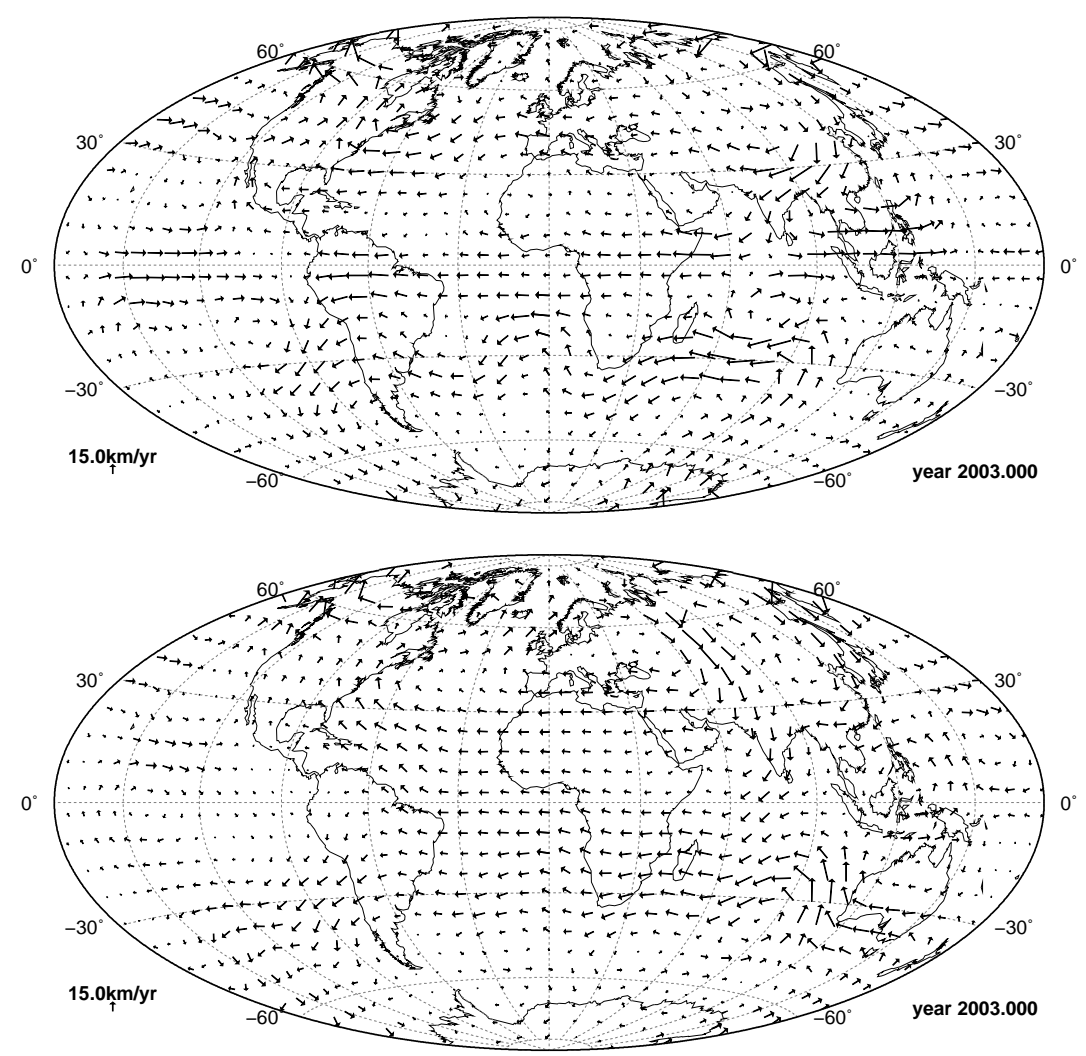

Fig. 6. Maps of the two flow solutions at the epoch 2003.: TG (top) and PT (bottom). The arrows describe the horizontal flow on the core surface, with the length indicating the magnitude and direction of the flow at certain positions. The continents are added as reference.

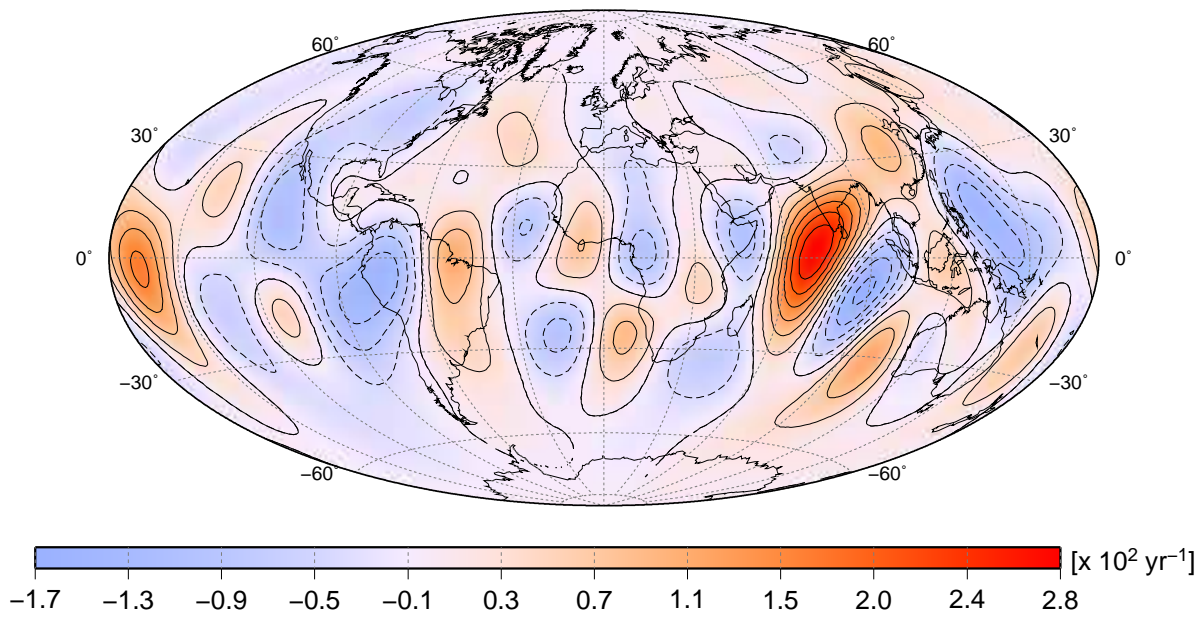

Fig. 7. Map of the fluid up- and downwelling, i.e. the horizontal divergence of the TG flow. 


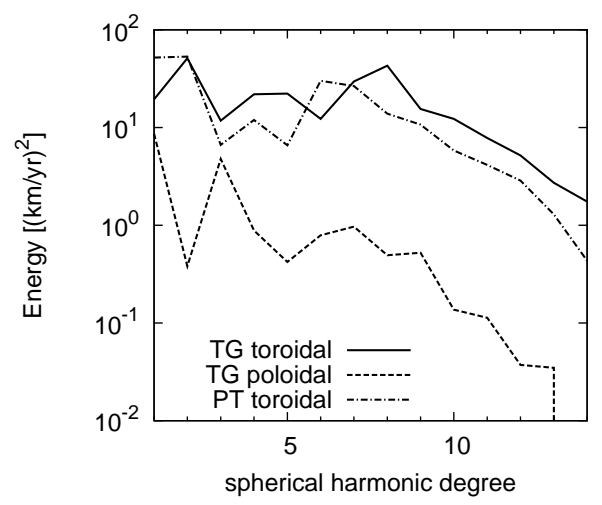

Fig. 8. Power spectra of the different flow constituents.

tures under the Indian ocean and the East Pacific. The finding of a strong TG motion under Indonesia is particularly interesting, because this region accommodates a large radial secular variation together with a weak radial field. We will discuss this further below.

The spectra of flow components (Fig. 8) show that both toroidal parts are very similar; however, the toroidal component of PT is slightly less energetic than that of TG for almost every degree. Again, this is due to the fact that the tangentially geostrophic assumption imposes a stronger constraint on the connections between the flow coefficients, and so the flow tends to have to be more energetic to fit the secular variation model. The power decreases with degrees above 8 and becomes minimal for higher degrees due to regularization. Note that the form of the misfit spectra are similar to those of [25], except for low degrees.

PT flow provide a slightly better fit to the secular variation model and observatory data than TG (cf. Table A.1). Also, the spectra of the misfit between observed and predicted secular variation, shown in Fig. 9, indicates that the misfit of TG is higher than of PT for almost all degrees. Both misfits exceed the secular variation in power at degrees 10 to 11. 


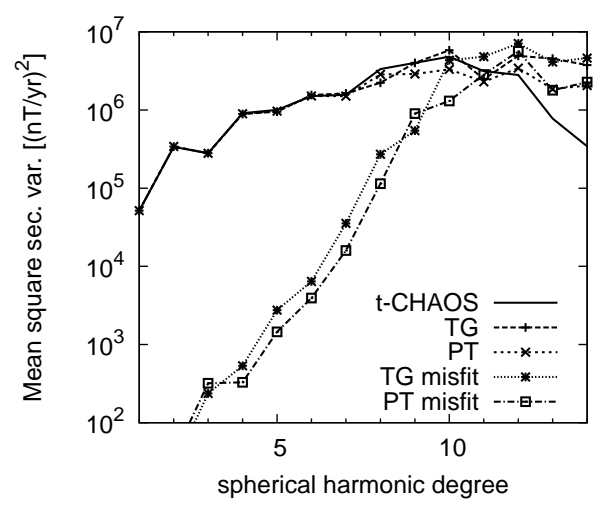

Fig. 9. Power spectra of the secular variation model, the predicted secular variation and the misfit at the CMB for the epoch 2003.

Ageostrophic motions are deviations from tangentially geostrophic balance and can be evaluated by computing $\nabla_{h} \cdot(\mathbf{v} \cos \theta)$. For the TG flow this is zero by definition and therefore the tangentially geostrophic modes combine $100 \%$ of the kinetic flow energy. This is essentially not the case for PT flow. Fig. 10 shows the toroidal ageostrophic flow, which becomes significant in PT, making up about $9 \%$ of the total flow energy (cf. Table A.1). This type of motion is expected to occur when the Coriolis force is no longer dominant and the geostrophic force balance becomes invalid - particularly in equatorial regions. This is particularly significant as the implementation of tangential geostrophy requires no equatorial crossing flow, despite the approximation not being valid there. However, the ageostrophic motion is also strong elsewhere, e.g. under Europe. We conclude that for a better fit to the radial secular variation of tCHAOS and observatories, the contribution of ageostrophic flows, e.g. equatorcrossing flows, is important. 


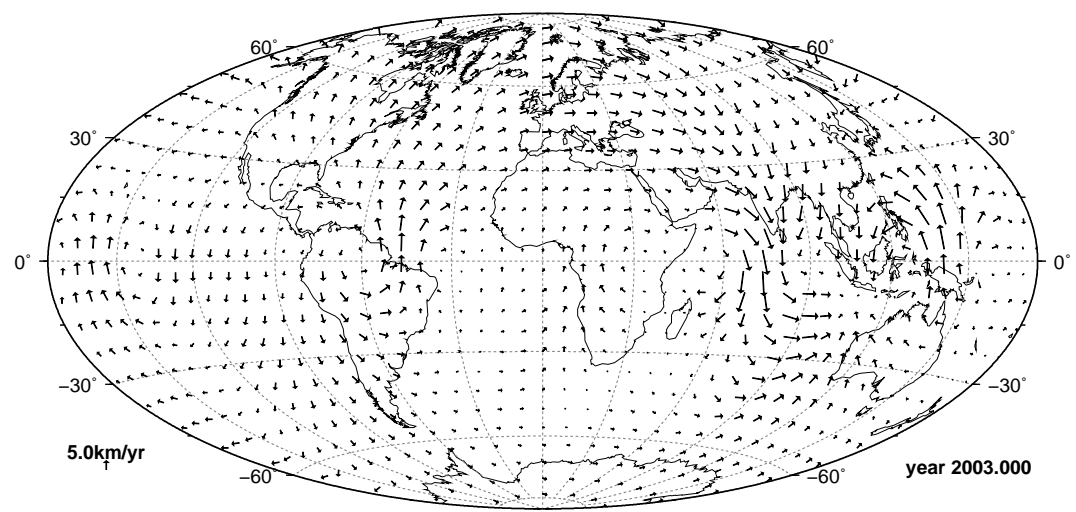

Fig. 10. Map of the toroidal ageostrophic motion of the purely toroidal fluid solution at the epoch 2003.

\subsection{Resolution analysis}

Resolution analysis allows estimation of the extent to which the flow is determined by the data, rather than by the regularization scheme. This may help to discriminate between the criteria of the flow solutions and their temporal behavior. The resolution matrix is given by

$$
\mathbf{R}=\left(\mathbf{A}^{\top} \mathbf{C}_{\mathbf{e}}{ }^{-1} \mathbf{A}+\mathbf{C}_{\mathbf{m}}{ }^{-1}\right)^{-1}\left(\mathbf{A}^{\top} \mathbf{C}_{\mathbf{e}}{ }^{-1} \mathbf{A}\right)
$$

where $\mathbf{C}_{\mathbf{e}}$ is defined in (8) and $\mathbf{C}_{\mathbf{m}}$ is the model covariance matrix as it is expected from the a-priori constraints on geostrophic, temporal and spatial smoothness of the model. The resolution matrix relates the expected to the actual model properties in the absence of data errors. Ideally, this matrix would be an identity matrix. Inadequacy of the data necessitates application of a regularization scheme to obtain a stable solution. A resolution near 1.0 signifies that a model parameter is wholly determined by the data, whereas a low resolution means that the model is mostly constrained by the a priori information, i. e. damping.

Fig. 11 show the diagonal elements of the resolution matrix of the toroidal and 

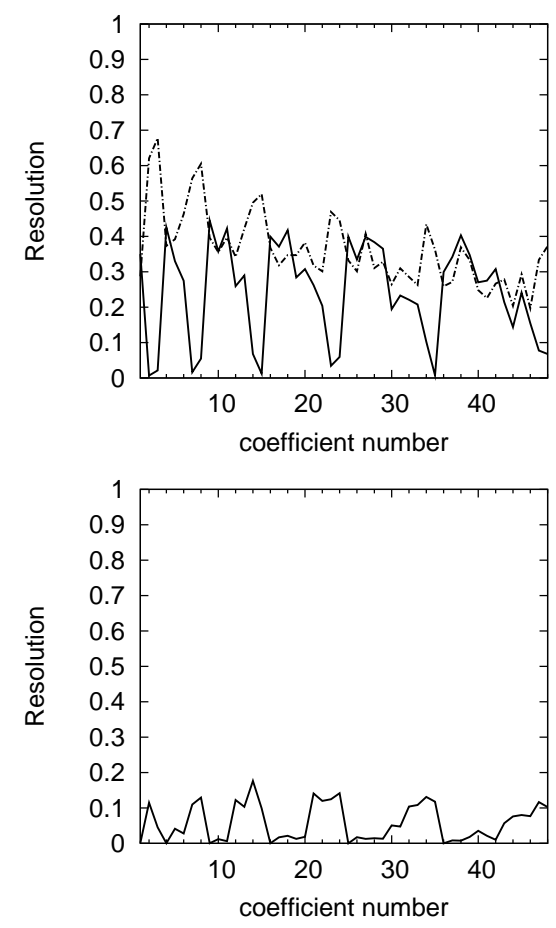

Fig. 11. The averaged resolution of the first 48 toroidal (top) and poloidal (bottom) flow coefficients are shown. A coefficient number of 48 corresponds to $t_{6}^{6}$ and $s_{6}^{6}$, respectively. TG coefficients (solid line) and PT coefficients (dashed line).

poloidal components for both flows. Generally, the toroidal flow coefficients are better resolved than the poloidal ones; resolution is non-zero for all degrees, whereas the resolution of the poloidal terms tends to zero above degree 10 . The resolutions of the toroidal components from PT and TG vary within a degree and are of comparable scale for the $t_{l}^{0}$ terms, but aside from that they are very different. The resolution of the TG toroidal terms has significant resolution for the zonal coefficients $\left(t_{l}^{0}\right.$, coefficient numbers $1,4,9,16,25$ and 36), whereas the resolutions of the sectorial terms $\left(t_{l}^{l}\right.$, coefficient numbers 2,3 , $7,8,14,15,23,24)$ are nearly zero. The near-zero resolution of the sectorial terms can be explained by the effect of the tangentially geostrophic constraint which restrains flow across the equator or perpendicular to the equator. The resolution of the PT toroidal terms does not vanish for sectorial terms, instead, 
the sectorial terms are the best resolved.

The resolution of the TG poloidal coefficients is only a fraction of the toroidal term resolution. The highest resolution is carried by the non-zonal terms where the coefficient $s_{4}^{1}$ has the maximum resolution. The complementary resolution of toroidal and poloidal terms is accounted for by the tangentially geostrophic constraint, which impose nothing on $t_{l}^{0}$ and strongly requires that $t_{l}^{l}$ and $s_{l}^{0}$ vanish.

The $s_{l}^{0}$ terms are required by tangential geostrophy to be zero, and therefore by definition are not resolved by the data. Interpretation of the other terms needs more care: a zero resolution does not mean that we have no information about their value. Tangential geostrophy imposes close correlations between coefficients. In these cases, a linear combination of coefficients including the "unresolved" coefficient is well-resolved, but the required correlation between those terms means that there is no independent determination of a coefficient, and so a zero-resolution as defined here.

\subsection{Uncertainties of the flow inversion}

In the previous section we discussed the results of the fluid flow inversion where it was assumed that the contribution of geomagnetic diffusion to the observed secular variation can be neglected. Here, we apply the same concept to estimate the effect of diffusion. In principle, the derived core surface flow provides a prediction of the radial secular variation at the CMB. Ideally, in the absence of geomagnetic diffusion, prediction and observation of the secular variation are equal. Deviations between the prediction and observation can 
arise from mainly three sources: I) the contributions of non-modelled secular variation produced by interaction of non-resolvable small-scale core flow with the core field [15], II) the inability to resolve the flow in the null-space of the radial induction equation, III) the disregard of magnetic diffusion.

In order to discuss the sources of residuals we derive the global distribution of the residuals at the core surface by

$$
\delta \dot{g}_{l}^{m}=\left(\frac{a}{c}\right)^{(l+2)} \frac{\dot{g}_{l}^{m}-\hat{\dot{g}}_{l}^{m}}{\sigma_{l}^{m}},
$$

and likewise $\delta \dot{h}_{l}^{m} \cdot \sigma_{l}^{m}=\sqrt{l+1}$ are the standard deviations of the Gauss coefficients and $\dot{g}_{l}^{m}$ and $\hat{\dot{g}}_{l}^{m}$ are the modelled secular variation coefficients and their predictions from the flow in $\mathrm{nT} / \mathrm{yr}$, respectively. $\delta \dot{g}_{l}^{m}$ and $\delta \dot{h}_{l}^{m}$ are used to compute the residuals of the radial component

$$
\delta \dot{\mathrm{B}}_{\mathrm{r}}=\sum_{l=1}^{14}(l+1) \sum_{m=0}^{l} P_{l}^{m}\left(\delta \dot{g}_{l}^{m} \cos m \phi+\delta \dot{h}_{l}^{m} \sin m \phi\right) .
$$

The normalization by the standard deviation prevents the residuals being dominated by the bluish power of the small-scale secular variation when downward continued to the core surface, since the same factor multiplies the coefficients and their errors and hence cancels. In Fig. 12 the absolute values of the radial residuals, instead of the radial residuals themselves, are shown in order to highlight large residuals.

In Fig. 12 red patches indicate regions where the inversion of the diffusionless induction equation fails to explain the observed secular variation. Both maps of Fig. 12 are similar and show several red patches in nearly the same regions. However, it should be noted that the residuals are less in amplitude for PT than for TG. Further, the residuals of TG tend to form patterns broadly perpendicular to the equator. It is interesting to note that similar patterns 

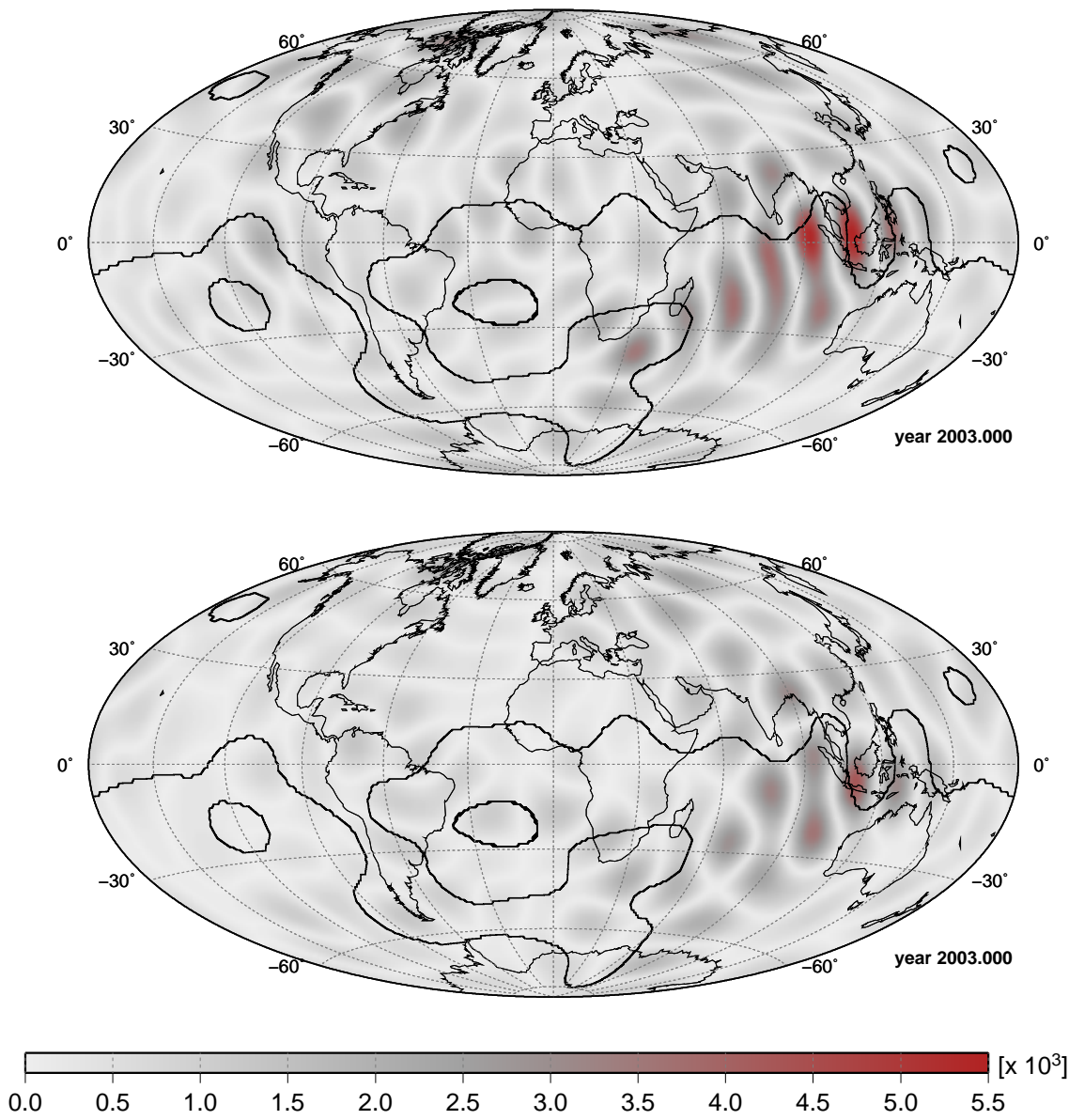

Fig. 12. Maps of the absolute radial residuals $\left|\delta \dot{\mathrm{B}}_{\mathrm{r}}\right|$ of TG (top panel) and PT (bottom panel) for 2003 . These maps are overlaid by contour lines of zero $B_{r}$. Caution should be paid to the fact that the absolute values are shown, which may simulate the features to be at small scale.

were discussed by [47], who also found large residuals under Indonesia and south-east Asia for the epochs 1970 and 1980, but using a different method to invert for the core surface flow.

Clearly, the regions of the largest residuals do not match with those found by [15] of maximal non-modelled secular variation. It is likely that contributions of non-modelled secular variation produced by interaction of non-resolvable small--scale core flow and the core field are not the cause of the residuals.

Are these residuals due to magnetic diffusion, due to the remaining non- 


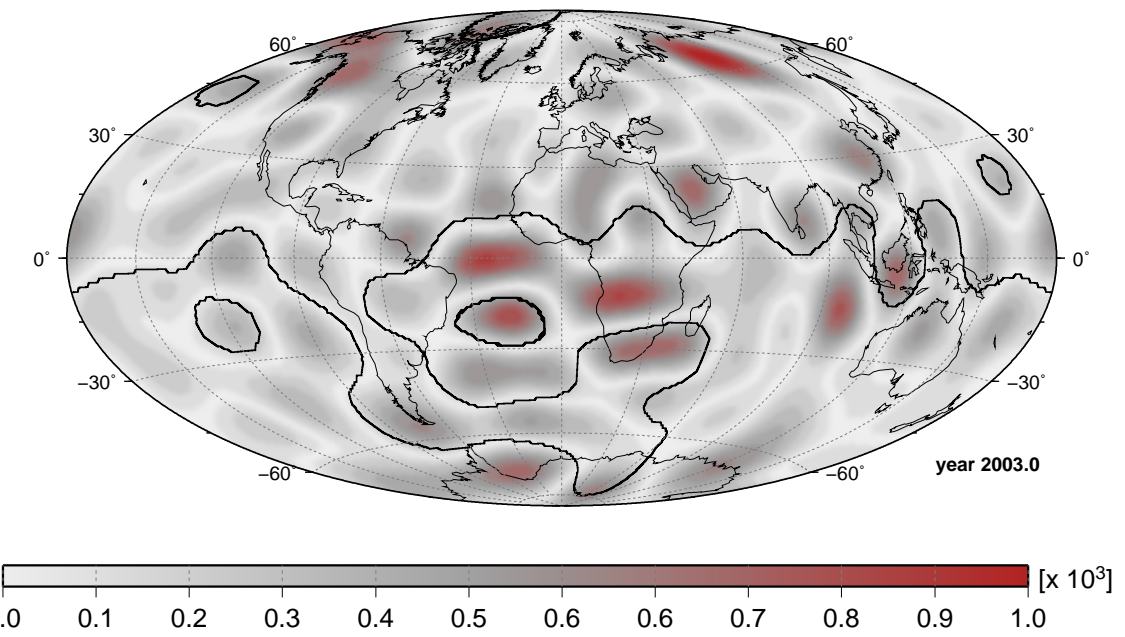

Fig. 13. Map of the absolute radial secular variation at the CMB due to field decay, as defined in (14).

uniqueness in the flow inversions, or are they artefacts of the modelling process? In order to quantify the effect of diffusion we follow [25], where the order of magnitude of the observed secular variation is estimated by considering diffusive free-decay modes applied to the CMB field. We denote this as static diffusion, because it does not invoke any motion. [20] has shown that the effect can be calculated by expanding the initial field in terms of decay modes. Then the resulting secular variation is

$$
\dot{\mathrm{B}}_{\mathrm{r}}(c) \sim \sum_{l, m} \sum_{n=1}^{3} B_{r l}^{m n}(c) \gamma_{l}^{n} / \sigma_{l}^{m}
$$

where $\gamma_{l}^{n}$ is the decay rate, proportional to the $n$th zero of the degree $l-1$ spherical Bessel function, taken from [36], which are independent of the order $m$. In this computation the first three zeros are used. In order to allow a comparison with the scale of the residuals the secular variation due to decay is also normalized by the standard deviation of the Gauss coefficients, $\sigma_{l}^{m}$.

The map of the static diffusion, Fig. 13, shows distinct features, some of them in regions where we expect the frozen flux hypothesis to be violated. Evidence for this has been noted under the southern Atlantic [10]. The patch under 


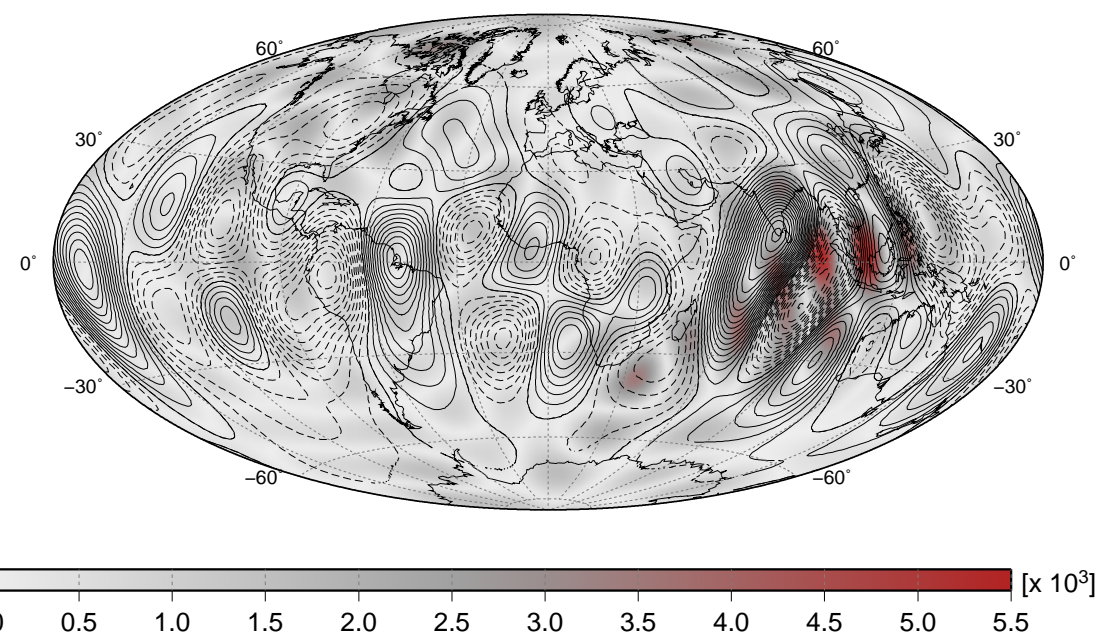

Fig. 14. Map of the absolute radial residuals overlaid by contours of the horizontal divergence of the flow velocity. The fluid fluid up- (solid lines) and downwelling (dashed lines) are also shown.

Ascension Island may be linked to variable magnetic flux of the reverse flux patch in that region. Other patches like the ones under Siberia and Alaska can be associated with the steady flux lobes. The patches north and west of Australia match closely the regions of the largest residuals. But, compared to the scale of Fig.12 static diffusion can only account for about $15 \%$ of the residuals. This is to much to rule out static diffusion as a part of the residuals. However, the conclusions to be drawn from this approach are to some extent ambiguous. First, because we cannot unambiguously indicate secular variation generated by either advection or diffusion, except for patches of reversed flux $[7 ; 10]$. Second, because moderate residuals are observed in the Southern Atlantic, and none of the red patches of Fig. 12 can be associated with such regions of flux expulsion. This does not imply that magnetic diffusion is absent in these regions, it simply may indicate that diffusion is fit by frozen-flux flow, although [38] found this scenario unlikely. To obtain realistic estimates of diffusive effects is demanding. Implementation of these computations, following [18], is in progress and will be reported separately. 
Despite the problem of quantifying the diffusive contributions to the residuals, we note again that the highest residuals appear in regions where weak radial field together with strong radial secular variation is observed. The tangentially geostrophic solution shows strong motion in these regions, whereas the purely toroidal flow is weak. In Fig. 14 the absolute residuals and the horizontal divergence of the TG flow are plotted. The quantity $\dot{B}_{r} / B_{r}$ is equal to the horizontal divergence of the flow and characterize up- and down-welling of the fluid flow at specific points [6]. This figure clearly identifies regions of high residuals with those of divergent and convergent flows, which indicates a flawed estimation of the poloidal flow components. The faulty derivation of the poloidal flow could be understood as a consequence of two facts: first, the expected failing of the tangentially geostrophic assumption in equatorially regions; and secondly, the tangentially geostrophic assumption imposes a stronger constraint on the poloidal than on the toroidal terms [37]. Most likely, a combination of the two causes leads to compromised estimation of the poloidal flow under the Indian Ocean and Indonesia. However, the failing of the tangential geostrophy in these regions to match the observed secular variation may not be utilized to explain the failing of PT in the same regions. Here, we can only speculate upon the causes.

\section{Temporal flow variability and the geomagnetic jerk}

In order to discuss possible causes of step-like changes in $\partial^{2} \mathrm{~B}_{\mathrm{r}} / \partial t^{2}$ (geomagnetic jerks), we have to consider the time derivative of the radial, diffusion-less 
induction equation. In doing so we write

$$
\begin{aligned}
\frac{\partial^{2} \mathrm{~B}_{\mathrm{r}}}{\partial t^{2}} & =-\nabla_{h} \cdot\left(\dot{\mathbf{v}} \mathrm{B}_{\mathrm{r}}\right)-\nabla_{h} \cdot\left(\mathbf{v} \dot{\mathrm{B}}_{\mathrm{r}}\right) \\
& =-\dot{\mathrm{B}}_{\mathrm{r}} \nabla_{h} \cdot \mathbf{v}-\mathrm{B}_{\mathrm{r}} \nabla_{h} \cdot \dot{\mathbf{v}}-\dot{\mathbf{v}} \cdot \nabla_{h} \mathrm{~B}_{\mathrm{r}}-\mathbf{v} \cdot \nabla_{h} \dot{\mathrm{B}}_{\mathrm{r}}
\end{aligned}
$$

The first two terms of the RHS invoke poloidal flow, which we believe is unimportant; therefore, these are not discussed further. The remaining two terms indicate that jumps in the secular acceleration are due to two processes. Evaluation of the fourth term in (15) is difficult. A simple interpretation would be that secular acceleration jumps can be generated by the action of the steady flow on temporally variable horizontal shear of the radial field. However, the evaluation of this effect is difficult, as $\nabla_{h} \cdot \dot{\mathrm{B}}_{\mathrm{r}}$ can also include diffusive processes which are poorly constrained.

We therefore concentrate on the third term of the RHS in (15), from which jumps can be caused by temporally variable flows acting on the horizontal gradient of the field (the third term of the RHS in (15)). In order to evaluate this mechanism the temporal variability of toroidal flow coefficients is examined, in part because TG and PT both provide estimates of the toroidal flow and a comparison is possible. Further, the geomagnetic jerk is mainly observed in the radial secular variation and (3) links the radial secular variation to the horizontal flow component. Thus, we expect the jerk signature to be clearest in the horizontal flow component.

In Fig. 15 we plot zonal flow coefficients vs. time. The changes are clearest in $t_{1}^{0}, t_{2}^{0}$ and $t_{3}^{0}$; the kinks in these terms coincide with or precede the jerk occurrence, suggesting a possible relation between changes in the large scale zonal flow and jerk. The coefficients $t_{4}^{0}$ show also changes in their slope, however, several other features appear which cannot be linked to the observed secular 
variation. The $t_{5}^{0}$ term of TG shows a kink in 2003.5 which may be related to the jerk in 2003. Other zonal flow coefficients, like $t_{6}^{0}, t_{7}^{0}, t_{8}^{0}$ and $t_{9}^{0}$ show no apparent relation to the geomagnetic jerk.

Changes in the equatorially-symmetric, toroidal zonal flow $t_{1}^{0}, t_{3}^{0}$ coincide with the occurrence time of the geomagnetic jerk in 2003; variations in these flow components are generally interpreted in terms of torsional oscillations. Sudden changes in the flow may occur, when the motion reaches its reversal points. Seemingly, this indicates a close relation between torsional oscillations and the occurrence of the geomagnetic jerk, as proposed by [9]. However, as pointed out by [16], zonal flow plays only a minor role in creating secular variation. Apart from the zonal flow coefficients some non-zonal terms show similar changes, such as $t_{1}^{1 s}, t_{3}^{1 s}$ and $t_{4}^{4}$, which supports the assumption that these terms are also important contributions to the jerk signal. Figure 16 shows the temporal change of the non-axissymmetric $t_{3}^{1}$ sine term. Another difficulty in explaining geomagnetic jerks in terms of torsional oscillations is the difference between typical timescale of torsional oscillations (of the order of several decades [12]) and the reoccurrence period of geomagnetic jerks (somewhat briefer than ten years). Moreover, there are indications that another jerk occurred around 2005 [35], suggesting an even shorter timescale of jerk occurrence. So, while a link between torsional oscillations and the 2003 geomagnetic jerk is indicated, torsional oscillations may not be the exclusive cause of it.

\section{Core angular momentum}

The Length-of-Day (LOD) is a measure of the rotation rate of the mantle; its variation provides information about the changes in the mantle moment 

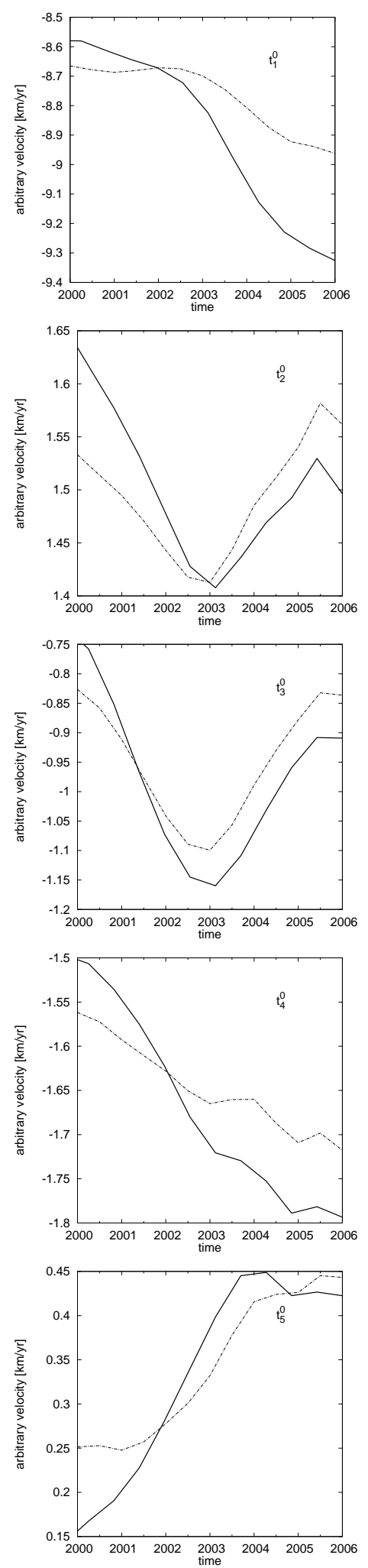

Fig. 15. The temporal variation of the first five zonal flow coefficients of the two flow solutions. To ease comparison, the coefficients of TG (solid line) are shifted to the range of the PT coefficients (dashed line). From top to bottom $t_{1}^{0}, t_{2}^{0}, t_{3}^{0}, t_{4}^{0}$ and $t_{5}^{0}$. 


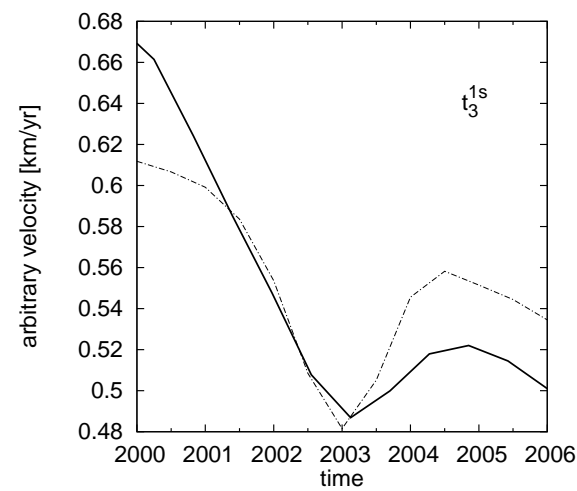

Fig. 16. The temporal variation of the non-axissymmetric $t_{3}^{1}$ sine flow coefficients of the two flow solutions, TG (solid line) and PT (dashed line), respectively.

of inertia or the axial torques acting on the mantle, from external or internal influences such as the dynamical coupling with the atmosphere or the Earth's core. A primary motivation to link geomagnetic jerks with variations in the fluid flow is given by the fact that geomagnetic jerks coincided with sudden changes in the LOD derivative [23]. [24; 34] provided strong evidence for a correlation between the 2003 jerk and changes in LOD. The relative core angular momentum (giving a prediction of changes in LOD) can be estimated from the core surface flow by a simple formulation which depends on two zonally symmetric toroidal flow components, $t_{1}^{0}$ and $t_{3}^{0}[27 ; 29]$. The flow predictions of the core angular momentum are compared to the observed LOD (Fig. 17). The LOD data used here are a rough fit to the observed LOD, where signals of atmospheric and oceanic angular momentum variation have been removed, following [23]. The general behavior of all three curves is similar, decreasing until 2003 followed by a rather flat section for the rest of the period. However, 


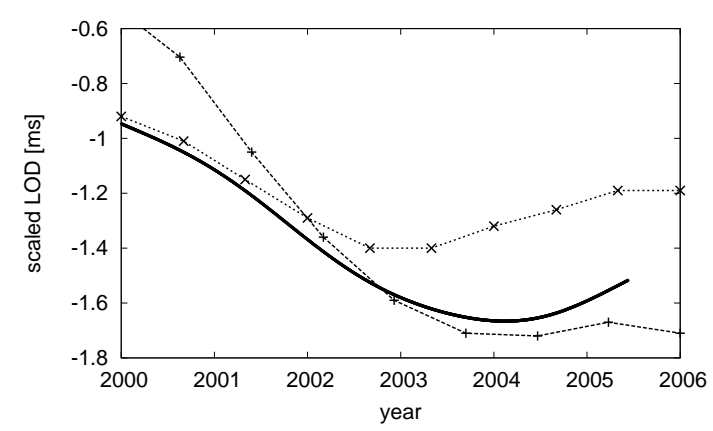

Fig. 17. Observed LOD variation (solid line) and its prediction from the two flow solutions: TG (solid line with + ) and PT (dashed line with $\mathrm{x}$ ).

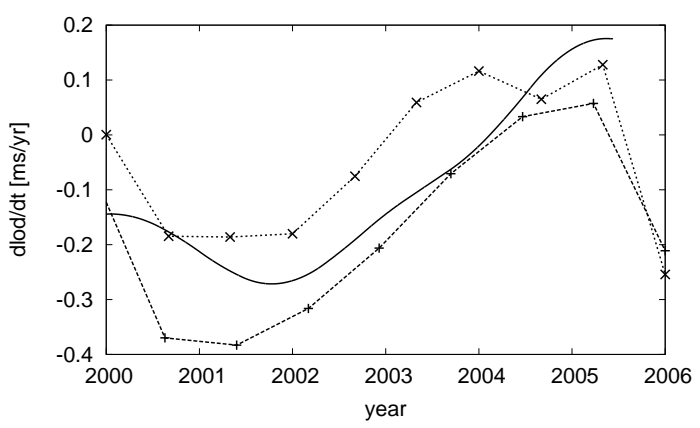

Fig. 18. First time derivatives of the observed LOD and the relative core angular momentum derived from TG and PT. Same line styles as in Fig. 17.

there are slight differences in the slope of the predicted curves.

A better diagnostic of the ability of the flow to predict $\triangle \mathrm{LOD}$ is given by a comparison of the first time derivative of the LOD and the core angular momentum, shown in figure 18. These time derivatives can be interpreted as torques acting between mantle and liquid core. Again, the shapes of the curves are in good agreement, with the tangentially geostrophic prediction fitting the observation particularly well, although amplitudes do not match perfectly. Additionally constraining the flows to fit observatory secular variation data does not provide a better fit to the observed LOD than the inversion based on t-CHAOS only. This is shown in Fig. 19, where the curve of a t-CHAOS based inversion closely matches one from a joint inversion of t-CHAOS and observa- 


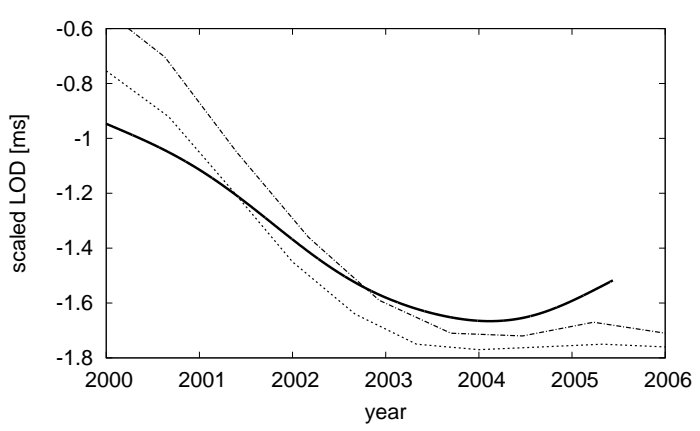

Fig. 19. Relative core angular momentum derived from the core surface flow: The prediction of the core surface flow when the flow is constrained to fit the observed secular variation at observatory locations (dash-dotted line) and the prediction without that constraint (dashed line). Both predictions are computed from tangentially geostrophic solutions with identical spatial and temporal damping. tory data. This result is surprising, because the secular variation is thought to be better resolved in time by observatory measurements than the t-CHAOS model (the improved fit to the observatory data from the flow model can be seen in Fig. 5). But it could be understood that for an accurate derivation of $t_{1}^{0}$ and $t_{3}^{0}$ a good global coverage of geomagnetic observatories is needed. The equally good fit from the satellite-data constrained model alone confirms the good temporal resolution of the secular variation in the t-CHAOS model.

\section{Conclusion}

In this study we have constructed continuous models of the fluid motion at the core surface between 2000 and 2006 which agree with the secular variation of the t-CHAOS model, to relate the geomagnetic jerk which occurred around 2003 to changes in the core surface flow. The major result of this study is that we find evidence for a coincidental occurrence of changes in the equatoriallysymmetric, toroidal zonal flow and the occurrence of the geomagnetic jerk 
in 2003. This flow is consistent with torsional oscillation and [9] promoted an explanation of jerks by torsional oscillation. However, we cannot confirm this hypothesis, because zonal flow plays a minor role in the secular variation generation [16] and other flows are of more effective in geomagnetic field changes.

The inversion of the diffusion-less induction equation fails in regions where we observe large secular variation in the radial field together with a weak radial field (cf. Fig. 2), i.e. under Indonesia. We identify two reasons, first the tangentially geostrophic constraint is expected to become invalid in equatorial regions. Second, the tangentially geostrophic constraint imposes a strong (and unphysical) constraint of no flow perpendicular to the equator. Further, the coincidence of large residuals and strong poloidal motion suggests that the tangentially geostrophic constrain leads to a compromised estimation of the poloidal flow component in the Indonesian region. The residuals significantly reduce when the purely toroidal flow assumption is used instead of tangential geostrophy. Apparently, the regions of largest residuals are not related to those where changes of the magnetic flux have been reported and diffusion is expected to act [10]. [24] pointed out that static diffusion may be relatively more important in flow modelling at low harmonic degree, and by this study we acquired an estimate that static diffusion accounts for $15 \%$ of the residuals of the flow models. Further analysis is needed in order to assign the possible contribution of diffusive processes to the residuals. Both flow assumptions provide equal estimates of the low degree equatorially-symmetric zonal flows, and therefore, their predictions of the relative core angular momentum match. These predictions show good agreement compared with the observed variation in $\triangle \mathrm{LOD}$. 


\section{Acknowledgement}

The authors thank Nils Olsen and Vincent Lesur for helpful discussions. The maps of the flow and radial secular variation are generated with GMT [45], all other plots are generated with gnuplot. 


\section{References}

[1] D. W. Allan and E. C. Bullard. Distortion of a toroidal field by convection. Rev. Mod. Physics, 30:1087-1088, 1958.

[2] G. E. Backus. Kinematics of geomagnetic secular variation in a perfectly conducting core. Philos. Trans. R. Soc. London A, 263:239-266, 1968.

[3] G. E. Backus. Non-uniqueness of the external geomagnetic field determined by surface intensity measurements. J. geophys. Res., 75:6337-6341, 1970.

[4] G. E. Backus. Determination of the external geomagnetic field from intensity measurements. Geophys. Res. Lett., 1:21, 1974.

[5] G. E. Backus and J.-L. Le Mouël. The region on the core-mantle boundary where a geostrophic velocity field can be determined from frozen flux magnetic data. Geophys. J. R. astr. Soc., 85:617-628, 1986.

[6] E. R. Benton. A simple method for determining the vertical growth rate of vertical motion at the top of Earth's outer core. Phys. Earth Planet. Inter., 24:242-244, 1981.

[7] J. Bloxham. The expulsion of magnetic flux from the Earth's core. Geophys. J. R. astr. Soc., 87:669-678, 1986.

[8] J. Bloxham. The determination of fluid flow at the core surface from geomagnetic observations. In N. J. Vlaar, G. Nolet, M. J. R. Wortel, and S. A. P. L. Cloetingh, editors, Mathematical Geophysics, A Survey of Recent Developments in Seismology and Geodynamics. Reidel, Dordrecht, 1988.

[9] J. Bloxham, M. Dumberry, and S. Zatman. The origin of geomagnetic jerks. Nature, 420:65-68, 2002.

[10] J. Bloxham and D. Gubbins. The secular variation of earth's magnetic 
field. Nature, 317:777-781, 1985.

[11] J. Bloxham and A. Jackson. Fluid flow near the surface of the Earth's outer core. Rev. Geophys., 29:97-120, 1991.

[12] S. I. Braginsky. Torsional magnetohydrodynamic vibrations in the Earth's core and variations in day length. Geomag. and Aeronomy (English translation), 10:1-8, 1970.

[13] S. I. Braginsky. Short-period geomagnetic secular variation. Geophys. Astrophys. Fluid Dyn., 30:1-78, 1984.

[14] D. W. Eaton and J.-M. Kendall. Improving seismic resolution of outermost core structure by multichannel analysis and deconvolution of broadband SmKS phases. Physics of the Earth and Planetary Interiors, 155:104-119, 2006.

[15] C. Eymin and G. Hulot. On core surface flows inferred from satellite magnetic data. Physics of the Earth and Planetary Interiors, 152:200$220,2005$.

[16] C. Gire and J.-L. Le Mouël. Tangentially geostrophic flow at the coremantle boundary compatible with the observed geomagnetic secular variation: The large-scale component of the flow. Phys. Earth Planet. Int., 59:259-287, 1990.

[17] D. Gubbins. Geomagnetic field analysis - II. Secular variation consistent with a perfectly conducting core. Geophys. J. R. astr. Soc., 77:753-766, 1984.

[18] D. Gubbins. A formalism for the inversion of geomagnetic data for core motions with diffusion. Phys. Earth Planet. Inter., 98:193-206, 1996.

[19] D. Gubbins. Geomagnetic constraints on stratification at the top of Earth's core. Earth Planets Space, 59:661-664, 2007.

[20] D. Gubbins and P. H. Roberts. Magnetohydrodynamics of the Earth's 
core. In J. A. Jacobs, editor, Geomagnetism, volume 2, chapter 1. Academic, San Diego, Calif., 1987.

[21] R. G. Hills. Convection in the Earth's mantle due to viscous shear at the core-mantle interface and due to large-scale buoyancy. PhD thesis, New Mexico State University, Las Cruces, 1979.

[22] R. Holme. Electromagnetic core-mantle coupling II: probing deep mantle conductance. In M. Gurnis, M. E. Wysession, E. Knittle, and B. A. Buffett, editors, The Core-Mantle Boundary Region, pages 139151. AGU,Washington DC., 1998.

[23] R. Holme and O. de Viron. Geomagnetic jerks and a high-resolution length-of-day profile for core studies. Geophys. J. Int., 160:435-440, 2005.

[24] R. Holme and O. de Viron. Evidence for a geomagnetic jerk after 2003 in LOD. In Proceedings of the First International Swarm Science Meeting, volume WPP-261. ESA, 2006.

[25] R. Holme and N. Olsen. Core surface flow modelling from high-resolution secular variation. Geophys. J. Int., 166:518-528, 2006.

[26] G. Hulot, C. Eymin, B. Langlais, M. Mandea, and N. Olsen. Small-scale structure of the geodynamo inferred from Ørsted and Magsat satellite data. Nature, 416:620-623, 2002.

[27] A. Jackson. Time-dependency of tangentially geostrophic core surface motions. Phys. Earth Planet. Inter., 103:293-311, 1997.

[28] A. Jackson, J. Bloxham, and D. Gubbins. Time-dependent flow at the core surface and conservation of angular momentum in the coupled coremantle system. In J.-L. Le Mouël, D. E. Smylie, and T. Herring, editors, Dynamics of the Earth's deep interior and Earth rotation, pages 97-107. AGU/IUGG, 1993.

[29] D. Jault, C. Gire, and J. L. Le Mouël. Westward drift, core motions 
and exchanges of angular momentum between core and mantle. Nature, 333:353-356, 1988.

[30] J.-L. Le Mouël. Outer core geostrophic flow and secular variation of Earth's magnetic field. Nature, 311:734-735, 1984.

[31] J.-L. Le Mouël, C. Gire, and T. Madden. Motions at the core surface in the geostrophic approximation. Phys. Earth Planet. Int., 39:270-287, 1985.

[32] T. Neubert, M. Mandea, G. Hulot, R. von Frese, F. Primdahl, J. L. Jørgensen, E. Friis-Christensen, P. Stauning, N. Olsen, and T. Risbo. Ørsted satellite captures high-precision geomagnetic field data. Eos Trans. AGU, 82:81-88, 2001.

[33] N. Olsen, H. Lühr, T. J. Sabaka, M. Mandea, M. Rother, L. TøffnerClausen, and S. Choi. CHAOS-a model of the Earth's magnetic field derived from CHAMP, Ørsted, and SAC-C magnetic satellite data. Geophysical Journal International, 166:67-75, 2006.

[34] N. Olsen and M. Mandea. Investigation of a secular variation impulse using satellite data: The 2003 geomagnetic jerk. Earth and Planetary Science Letters, 255:94-105, 2007.

[35] N. Olsen and M. Mandea. Rapidly changing flows in the Earth's core. submitted to Science, 2007.

[36] F.J. Olver. Bessel Functions Part III: Zeros and associated values, volume 7 of Royal Society Mathematical Tables. Cambridge Univesity Press, Cambridge, UK, 1960.

[37] A. Pais and G. Hulot. Length of day decade variations, torsional oscillations and inner core superrotation: Evidence from recovered core surface zonal flows. Phys. Earth Planet. Inter., 118:291-316, 2000.

[38] S. Rau, U. Christensen, A. Jackson, and J. Wicht. Core flow inversions 
tested with numerical dynamo models. Geophys. J. Int., 141:485-497, 2000.

[39] C. Reigber, H. Lühr, and P. Schwintzer. CHAMP mission status. Adv. Space Res., 30:129-134, 2002.

[40] P. H. Roberts and S. Scott. On the analysis of secular variation, 1, A hydromagnetic constraint: Theory. J. Geomag. Geoelectr., 17:137-151, 1965.

[41] J. B. Taylor. The magneto-hydrodynamics of a rotating fluid and the Earth's dynamo problem. Philos. Trans. R. Soc. London Ser. A, 274:274$283,1963$.

[42] E. H. Vestine and A. B. Kahle. The Small Amplitude of Magnetic Secular Change in the Pacific Area. J. geophys. Res., 71:527, 1966.

[43] C. V. Voorhies. Narrow-scale flow and a weak field by the top of Earth's core: Evidence from Ørsted, Magsat, and secular variation. J. geophys. Res., 109:B03106, 2004.

[44] I. Wardinski and R. Holme. A time-dependent model of the earth's magnetic field and its secular variation for the period 1980 to 2000. J. geophys. Res., 111:12101, 2006.

[45] P. Wessel and W. H. F. Smith. Free software helps map and display data. Eos Trans. AGU, 72:445-446, 1991.

[46] K. A. Whaler. Does the whole of the Earth's core convect? Nature, 287:528-530, 1980.

[47] K. A. Whaler. Properties of steady flows at the core-mantle boundary in the frozen-flux approximation. Phys. Earth Planet. Inter., 68:144-155, 1991.

[48] S. Zatman and J. Bloxham. Torsional oscillations and the magnetic field within the Earth's core. Nature, 388:760-763, 1997. 
[49] S. Zatman and J. Bloxham. A one-dimensional map of $B_{S}$ from torsional oscillations of the Earth's core. In M. Gurnis, M. E. Wysession, E. Knittle, and B. A. Buffett, editors, The Core-Mantle Boundary Region, pages 183196. AGU, 1998. 


\section{A Appendix}

This table contains the damping parameter and some characteristics of the two flow solutions. 
Table A.1

Damping parameters and diagnostics of the two flow solutions

\begin{tabular}{lrr} 
Model parameter and diagnostics & TG & PT \\
\hline Damping parameters & $10^{6}$ & 0.00 \\
$\lambda_{g}$ & $2 \times 10^{-6}$ & $2 \times 10^{-6}$ \\
$\lambda_{s}$ & 10 & 10 \\
$\lambda_{t}$ & $1.3 \times 10^{-7}$ & 0.0 \\
\hline Geostrophic norm & $25.6 \times 10^{6}$ & $14.1 \times 10^{6}$ \\
Spatial smoothness norm & 3.4 & 2.8 \\
Temporal smoothness norm & 17.8 & 16.4 \\
Mean velocity (km/yr) & 2.51 & 2.04 \\
rms secular variation misfit (nT/yr) & 0.74 & 0.72 \\
rms misfit to observatory data & 365 & 393 \\
\hline
\end{tabular}

Relative to the total flow energy

\begin{tabular}{lrr}
\hline toroidal component & $93.3 \pm 0.09$ & $100.0 \pm 0.00$ \\
poloidal component & $6.7 \pm 0.09$ & $0.0 \pm 0.00$ \\
geostrophic component & $100.0 \pm 0.00$ & $91.3 \pm 0.18$ \\
ageostrophic component & $0.0 \pm 0.00$ & $8.7 \pm 0.17$ \\
symmetric component & $72.5 \pm 0.42$ & $77.2 \pm 0.53$ \\
symmetric toroidal component & $72.1 \pm 0.43$ & $77.2 \pm 0.53$ \\
symmetric poloidal component & $0.4 \pm 0.01$ & $0.0 \pm 0.00$ \\
asymmetric component & $27.5 \pm 0.42$ & $22.8 \pm 0.58$ \\
asymmetric toroidal component & $21.2 \pm 0.49$ & $22.8 \pm 0.53$ \\
asymmetric poloidal component & $6.3 \pm 0.09$ & $0.0 \pm 0.00$
\end{tabular}

